\title{
The Synergistic Effect of Functional Status and Comorbidity Burden on Mortality: A 16-Year Survival Analysis
}

\author{
Cynthia Chen ${ }^{1}$, Isaac Sia ${ }^{2}$, Hon-ming $\mathrm{Ma}^{3}$, Bee Choo Tai ${ }^{1}$, Angela Cheong ${ }^{1}$, Ngan Phoon Fong ${ }^{1}$, Shi Yu \\ Julia Tan ${ }^{4}$, Kin Ming Chan ${ }^{5}$, Boon Yeow Tan ${ }^{6}$, Edward Menon ${ }^{7}$, Chye Hua Ee ${ }^{8}$, Kok Keng Lee ${ }^{8}$, \\ Yee Sien $\mathrm{Ng}^{9}$, Yik Ying Teo ${ }^{1,10,11,12}$, Stefan $\mathrm{Ma}^{13}$, Derrick Heng ${ }^{13}$, Gerald Choon-Huat Koh ${ }^{1,4 *}$
}

1 Saw Swee Hock School of Public Health, National University of Singapore, National University Health System, Singapore, Singapore, 2 College of Public Health and Health Professions, University of Florida, Gainesville, Florida, United States of America, 3 Departments of Medicine and Therapeutics, Prince of Wales Hospital, The Chinese University of Hong Kong, Hong Kong SAR, China, $\mathbf{4}$ Yong Loo Lin School of Medicine, National University of Singapore, National University Health System, Singapore, Singapore, 5 Medical Services, Ang Mo Kio Thye Hua Kwan Hospital, Singapore, Singapore, 6 Medical Services, St Luke's Hospital, Singapore, Singapore, 7 Medical Services, St Andrew's Community Hospital, Singapore, Singapore, 8 Bright Vision Hospital, Singapore, Singapore, 9 Department of Rehabilitation Medicine, Singapore General Hospital, Singapore, Singapore, $\mathbf{1 0}$ Genome Institute of Singapore, Agency for Science, Technology, and Research, Singapore, Singapore, $\mathbf{1 1}$ Graduate School for Integrative Science and Engineering, National University of Singapore, Singapore, Singapore, 12 Department of Statistics and Applied Probability, National University of Singapore, Singapore, Singapore, 13 Ministry of Health, Singapore, Singapore

\begin{abstract}
Objectives: The relationship between disability and comorbidity on mortality is widely perceived as additive in clinical models of frailty.

Design: National data were retrospectively extracted from medical records of community hospital.

Data Sources: There were of 12,804 acutely-disabled patients admitted for inpatient rehabilitation in Singapore rehabilitation community hospitals from 1996 through 2005 were followed up for death till 31 December 2011.

Outcome Measure: Cox proportional-hazards regression to assess the interaction of comorbidity and disability at discharge on all-cause mortality.

Results: During a median follow-up of 10.9 years, there were 8,565 deaths (66.9\%). The mean age was 73.0 (standard deviation: 11.5) years. Independent risk factors of mortality were higher comorbidity $(p<0.001)$, severity of disability at discharge $(p<0.001)$, being widowed (adjusted hazard ratio [aHR]: 1.38, 95\% confidence interval [CI]:1.25-1.53), low socioeconomic status (aHR:1.40,95\%Cl:1.29-1.53), discharge to nursing home (aHR:1.14, 95\%Cl:1.05-1.22) and re-admission into acute care (aHR:1.54, 95\%Cl:1.45-1.65). In the main effects model, those with high comorbidity had an aHR=2.41 $(95 \% \mathrm{Cl}: 2.13-2.72)$ whereas those with total disability had an $\mathrm{aHR}=2.28(95 \% \mathrm{Cl}: 2.12-2.46)$. In the interaction model synergistic interaction existed between comorbidity and disability $(p<0.001)$ where those with high comorbidity and total disability had much higher aHR=6.57 (95\%Cl:5.15-8.37).

Conclusions: Patients with greater comorbidity and disability at discharge, discharge to nursing home or re-admission into acute care, lower socioeconomic status and being widowed had higher mortality risk. Our results identified predictive variables of mortality that map well onto the frailty cascade model. Increasing comorbidity and disability interacted synergistically to increase mortality risk.
\end{abstract}

Citation: Chen C, Sia I, Ma H-m, Tai BC, Cheong A, et al. (2014) The Synergistic Effect of Functional Status and Comorbidity Burden on Mortality: A 16-Year Survival Analysis. PLoS ONE 9(8): e106248. doi:10.1371/journal.pone.0106248

Editor: Jerson Laks, Federal University of Rio de Janeiro, Brazil

Received April 1, 2014; Accepted July 29, 2014; Published August 29, 2014

Copyright: (C) 2014 Chen et al. This is an open-access article distributed under the terms of the Creative Commons Attribution License, which permits unrestricted use, distribution, and reproduction in any medium, provided the original author and source are credited.

Data Availability: The authors confirm that all data underlying the findings are fully available without restriction. All relevant data are within the paper and its Supporting Information files.

Funding: This study was supported by a National University of Singapore (NUS) Start-Up Grant, the NUS Provost Matching Grant, the NUS Academic Research Fund, Ministry of Health (Singapore) Health Services Research Competitive Research Grant Number HSRG/0006/2013, Singapore National Medical Research Council Research Fellowship and Schulich Graduate Scholarship, University of Western Ontario. The funders had no role in study design, data collection and analysis, decision to publish, or preparation of the manuscript.

Competing Interests: The authors have declared that no competing interests exist.

*Email: gerald_koh@nuhs.edu.sg 


\section{Introduction}

Frailty is an important clinical syndrome that increases an individual's vulnerability to adverse health outcomes and mortality. [1-3] The classic frailty cascade model describes how agerelated physiological deterioration, stressful events, functional decline, recovery, disability, hospitalization, and institutionalization ultimately contribute to premature death in older individuals. [4] Older patients admitted for acute illness may have multiple chronic conditions, [5] experience disability,[6] and become institutionalized following discharge from the acute care setting. [7] Development of physical frailty in older individuals is greatly impacted by age-related physiological deterioration and a wide range of diseases and medical conditions. [8,9] Comorbidity is the concurrent presence of two or more medically diagnosed diseases, [3] and is commonly experienced by older individuals. [8-11] Comorbidity has also been shown to increase the risk of premature death in a variety of patients such as breast cancer, [12] essential hypertension or diabetes[13] and spinal cord injury. [14] Disability can be characterized as dependency in performing activities of daily living[3] and are common in older individuals. Elderly patients are particularly vulnerable to disability and functional decline after acute hospitalizations due to enforced immobilization and deconditioning.[3,5,15] Development of disabilities in older patients results in not only substantial costs, but also increased long term morbidity and mortality.[16-18] For example, hip fracture may reduce life expectancy by as much as $25 \%$.[16]

Some authors have perceived comorbidity as an etiologic risk factor and disability as an outcome of frailty.[19,20] Other models of frailty such as the frailty cascade,[4] Frailty Index (FI) [21,22] and Frailty Scale (FS)[23,24] include both disability and comorbidity as risk factors of premature mortality outcome.[25,26] Although relationship between frailty and mortality has been shown previously, $[27,28]$ the relative and combined impact of specific aspects of frailty (e.g. comorbidity and disability) remain unclear. The specific aims of this study were to identify predictors of mortality and to describe the combined effect of comorbidity and disability on mortality with reference to models of frailty.

\section{Methods}

\section{Study Population}

The study population was a historical national cohort taken from a database of all patients admitted to all community hospitals in Singapore between 1996 and 2005. Community hospitals are rehabilitation hospitals that cater to patients who are fit for discharge from acute hospitals but require inpatient convalescent or subacute rehabilitative care before returning to a final domiciliary site.[29] We excluded patients who died during their hospital stay in the community hospital $(n=24)$. Inclusion criteria for this study were:

1. First admission to community hospitals for inpatient rehabilitation from acute disability (e.g. stroke and hip fractures); and

2. Disability assessed at discharge.

The study was approved by the National University of Singapore Institutional Review Board (NUS-IRB) and ethics committees of Ang Mo Kio Thye Hua Kwan Hospital, Bright Vision Hospital, St Andrew's Community Hospital and St Luke's Hospital. Written informed consent of the patient was waived by approving NUS-IRB. The corresponding author and all research nurses have taken the oath of confidentiality under Singapore's
Official Secrets Act and only the minimum number of research personnel had access to the de-identified dataset.

\section{Data Extraction}

Trained research nurses performed data extraction from noncomputerized medical records between November 2005 and August 2008. Multiple iterations of data cleaning and verification were performed. An independent physician analyzed a $10 \%$ random sample of patients for data extraction accuracy and the error rate was $0.07 \%$.

\section{Covariates}

A number of covariates were controlled to elicit the specific effects of comorbidity and disability including socio-demographic variables such as age group (18-64, $\geq 65$ years), gender (female, male), ethnicity (Chinese, Malay, Indian, others), marital status (single, married, widowed, separated or divorced), caregiver availability (no, yes) and length of hospital stay. Hospitalization subsidy level served as a proxy measure for socioeconomic status (SES). In Singapore, $81 \%$ of public hospitals' beds (B2 and C class) are highly subsidized.[29] B2+ and B1 class lower subsidy and A class have no subsidy. For this study, SES was classified into three groups: high SES (A, B1 and B2+ class), moderate SES (B2 class) and low SES ( $\mathrm{C}$ class). Discharge destinations of patients were home $(\mathrm{n}=9,774)$, acute hospital $(\mathrm{n}=1,583)$, nursing home $(\mathrm{n}=1,251)$ and others $(\mathrm{n}=196)$. As the numbers who were discharged to other destinations were low, they were excluded from multivariate models.

\section{Exposures of interest}

Comorbidity. Comorbidity was measured using the Charlson comorbidity index (CCI).[12] Comorbidity data was manually extracted by trained nurses by reviewing the patients' medical problem list and complete medical records. The CCI measures the burden of medical illnesses and comprises of 19 different categories. The overall CCI score is a summation of weighted scores. A higher score reflects greater cumulative disease burden. We categorized index scores into four groups: no comorbidity $(0)$, low comorbidity (1-3), moderate comorbidity (4-6) and high comorbidity (7-16). The Charlson comorbidity score also has been shown to have a high inter-rater reliability of a kappa score of 0.93. [30]

Disability at discharge. As recommended by the Singapore Ministry of Health guidelines, disability was assessed in both community hospitals at admission and discharge[29] using the Shah modified Barthel Index (BI). This is usually done by qualified physiotherapists and/or occupational therapists in all community hospitals. The BI is a reliable and widely-used assessment for functional status [31] and disability.[32,33] It comprises of 10 items measuring distinct activities of daily living (ADL). The BI utilizes a five-point scale for each $\mathrm{ADL}$ and has a maximum score of 100. [34] BI scores are categorized by the severity of disability: 024 (total disability), 25-49 (severe disability), 50-74 (moderate disability), 75-90 (mild disability), 91-99 (minimal disability) and 100 (no disability).[34] For this study, the last three categories were collapsed into one category (75-100) and termed "no or mild disability" because of small sample sizes. The reliability of the BI has been demonstrated in numerous studies and test-retest, intrarater and inter-rater reliability have been shown to be high by correlation methods $(\mathrm{r}=0.87,0.71-0.99$ and $0.75-0.99$ respectively).[35,36] We chose to use discharge BI score of patients admitted for rehabilitation because this represented the best functional status after optimization with therapy. 
Table 1. Social demographics by discharge disability.

\begin{tabular}{|c|c|c|c|c|c|c|}
\hline & $\begin{array}{l}\text { Total } \\
(n=12,804)\end{array}$ & $\begin{array}{l}\text { No or mild disability } \\
(\mathrm{n}=\mathbf{5 0 8 2})\end{array}$ & $\begin{array}{l}\text { Moderate disability } \\
(\mathrm{n}=\mathbf{3 7 5 0})\end{array}$ & $\begin{array}{l}\text { Severe disability } \\
(n=1886)\end{array}$ & $\begin{array}{l}\text { Total disability } \\
(n=2086)\end{array}$ & P-value $^{a}$ \\
\hline \multicolumn{7}{|l|}{ Year of admission } \\
\hline 1996 & $585(4.6)$ & $116(2.3)$ & $100(2.7)$ & $60(3.2)$ & $309(14.8)$ & $<0.001$ \\
\hline 1997 & $1291(10.1)$ & $348(6.9)$ & $384(10.2)$ & $196(10.4)$ & $363(17.4)$ & \\
\hline 1998 & $1316(10.3)$ & $382(7.5)$ & $409(10.9)$ & $245(13.0)$ & $280(13.4)$ & \\
\hline 1999 & $1357(10.6)$ & $571(11.2)$ & $429(11.4)$ & $196(10.4)$ & $161(7.7)$ & \\
\hline 2000 & $1205(9.4)$ & $519(10.2)$ & $374(10.0)$ & $175(9.3)$ & $137(6.6)$ & \\
\hline 2001 & $1239(9.7)$ & $512(10.1)$ & $371(9.9)$ & $194(10.3)$ & $162(7.8)$ & \\
\hline 2002 & $1113(8.7)$ & $462(9.1)$ & $365(9.7)$ & $141(7.5)$ & $145(7.0)$ & \\
\hline 2003 & $1368(10.7)$ & $552(10.9)$ & $379(10.1)$ & $224(11.9)$ & $213(10.2)$ & \\
\hline 2004 & $1631(12.7)$ & $800(15.7)$ & $450(12.0)$ & $228(12.1)$ & $153(7.3)$ & \\
\hline 2005 & $1699(13.3)$ & $820(16.1)$ & $489(13.0)$ & $227(12.0)$ & $163(7.8)$ & \\
\hline \multicolumn{7}{|l|}{ Primary diagnosis at admission } \\
\hline Stroke & $5240(40.9)$ & 1557 (30.6) & $1536(41.0)$ & $880(46.7)$ & $1267(60.7)$ & $<0.001$ \\
\hline Fracture & $3781(29.5)$ & $1733(34.1)$ & $1188(31.7)$ & 464 (24.6) & $396(19.0)$ & \\
\hline Amputation & $299(2.3)$ & $135(2.7)$ & $98(2.6)$ & $29(1.5)$ & $37(1.8)$ & \\
\hline Others & $2434(19.0)$ & $1110(21.8)$ & $663(17.7)$ & $375(19.9)$ & $286(13.7)$ & \\
\hline Lower limb arthroplasty & $362(2.8)$ & $257(5.1)$ & $75(2.0)$ & $20(1.1)$ & $10(0.5)$ & \\
\hline Cancer & $243(1.9)$ & $113(2.2)$ & $66(1.8)$ & $39(2.1)$ & $25(1.2)$ & \\
\hline Falls & $232(1.8)$ & $104(2.1)$ & $77(2.1)$ & $31(1.6)$ & $20(1.0)$ & \\
\hline Pneumonia & $213(1.7)$ & $73(1.4)$ & $47(1.3)$ & $48(2.6)$ & $45(2.2)$ & \\
\hline \multicolumn{7}{|l|}{ Gender } \\
\hline Female & $7463(58.3)$ & $2934(57.7)$ & $2302(61.4)$ & $1057(56.0)$ & $1170(56.1)$ & $<0.001$ \\
\hline Male & $5341(41.7)$ & $2148(42.3)$ & $1448(38.6)$ & $829(44.0)$ & $916(43.9)$ & \\
\hline \multicolumn{7}{|l|}{ Age group } \\
\hline Age: $18-64$ years & $2671(20.9)$ & $1359(26.7)$ & $667(17.8)$ & $289(15.3)$ & $356(17.1)$ & $<0.001$ \\
\hline Age: $\geq 65$ years & $10133(79.1)$ & $3723(73.3)$ & $3083(82.2)$ & $1597(84.7)$ & $1730(82.9)$ & \\
\hline Length of stay (days), median $\left(25^{\text {th }}-75^{\text {th }}\right)$ & $32(20-47)$ & $32(22-47)$ & $33(23-47)$ & $33(21-46)$ & $33(19-49)$ & 0.053 \\
\hline \multicolumn{7}{|l|}{ Community hospital } \\
\hline A & $3240(25.3)$ & $1750(34.4)$ & $755(20.1)$ & $416(22.1)$ & $319(15.3)$ & $<0.001$ \\
\hline B & $6727(52.5)$ & $2591(51)$ & $2349(62.6)$ & $1115(59.1)$ & $672(32.2)$ & \\
\hline C & $2381(18.6)$ & $578(11.4)$ & $513(13.7)$ & $301(16.0)$ & $989(47.4)$ & \\
\hline D & 456 (3.6) & $163(3.2)$ & $133(3.6)$ & $54(2.9)$ & $106(5.1)$ & \\
\hline \multicolumn{7}{|l|}{ Ethnicity } \\
\hline Chinese & $11293(88.2)$ & $4578(90.1)$ & 3315 (88.4) & $1617(85.7)$ & $1783(85.5)$ & $<0.001$ \\
\hline Malay & $848(6.6)$ & $234(4.6)$ & $264(7.0)$ & $167(8.9)$ & $183(8.8)$ & \\
\hline Indian & $518(4.1)$ & $206(4.1)$ & $134(3.6)$ & $82(4.4)$ & $96(4.6)$ & \\
\hline Others & $145(1.1)$ & $64(1.3)$ & $37(1.0)$ & $20(1.1)$ & $24(1.2)$ & \\
\hline \multicolumn{7}{|l|}{ Marital status } \\
\hline Single & $1099(8.6)$ & $675(13.3)$ & $232(6.2)$ & $86(4.6)$ & $106(5.1)$ & $<0.001$ \\
\hline Married & $5483(42.8)$ & $2080(40.9)$ & $1594(42.5)$ & $849(45.0)$ & $960(46.0)$ & \\
\hline Widowed & $5853(45.7)$ & $2134(42)$ & $1826(48.7)$ & 917 (48.6) & $976(46.8)$ & \\
\hline Separated/Divorced & $369(2.9)$ & $193(3.8)$ & $98(2.6)$ & $34(1.8)$ & $44(2.1)$ & \\
\hline \multicolumn{7}{|l|}{ Caregiver } \\
\hline No & $1257(9.8)$ & $744(14.6)$ & $294(7.8)$ & $110(5.8)$ & $109(5.2)$ & $<0.001$ \\
\hline Yes & $11547(90.2)$ & $4338(85.4)$ & $3456(92.2)$ & $1776(94.2)$ & 1977 (94.8) & \\
\hline \multicolumn{7}{|l|}{ Socioeconomic status } \\
\hline High & $1207(9.4)$ & $424(8.3)$ & $445(11.9)$ & $178(9.4)$ & $160(7.7)$ & $<0.001$ \\
\hline Moderate & $4478(35.0)$ & $1480(29.1)$ & $1242(33.1)$ & $630(33.4)$ & $1126(54.0)$ & \\
\hline Low & 7119 (55.6) & $3178(62.5)$ & $2063(55.0)$ & $1078(57.2)$ & $800(38.4)$ & \\
\hline
\end{tabular}


Table 1. Cont.

\begin{tabular}{|c|c|c|c|c|c|c|}
\hline & $\begin{array}{l}\text { Total } \\
(n=12,804)\end{array}$ & $\begin{array}{l}\text { No or mild disability } \\
(n=5082)\end{array}$ & $\begin{array}{l}\text { Moderate disability } \\
(n=3750)\end{array}$ & $\begin{array}{l}\text { Severe disability } \\
(n=1886)\end{array}$ & $\begin{array}{l}\text { Total disability } \\
(n=2086)\end{array}$ & P-value $^{a}$ \\
\hline \multicolumn{7}{|c|}{ Comorbidity burden (Charlson comorbidity index) } \\
\hline None (0) & $2377(18.6)$ & $1344(26.5)$ & $652(17.4)$ & $196(10.4)$ & $185(8.9)$ & $<0.001$ \\
\hline Low (1-3) & $5878(45.9)$ & $2348(46.2)$ & $1742(46.5)$ & $864(45.8)$ & $924(44.3)$ & \\
\hline Moderate (4-6) & $4012(31.3)$ & $1217(24)$ & $1202(32.1)$ & $713(37.8)$ & $880(42.2)$ & \\
\hline High $(\geq 7)$ & $537(4.2)$ & $173(3.4)$ & $154(4.1)$ & $113(6.0)$ & $97(4.7)$ & \\
\hline \multicolumn{7}{|c|}{ Discharge destination } \\
\hline Home & $9774(77.5)$ & $4466(88.8)$ & $2844(77.3)$ & $1215(65.6)$ & $1249(61.1)$ & $<0.001$ \\
\hline Nursing Home & $1251(9.9)$ & $310(6.2)$ & $397(10.8)$ & $267(14.4)$ & $277(13.6)$ & \\
\hline Acute hospital & $1583(12.6)$ & $255(5.1)$ & $440(12.0)$ & $369(19.9)$ & $519(25.4)$ & \\
\hline
\end{tabular}

ap-value: Chi-square test for categorical variables and Kruskal-Wallis test for length of stay.

doi:10.1371/journal.pone.0106248.t001

\section{Determination of Endpoint}

The primary endpoint was mortality. Time-to-event was calculated from the time of discharge disability assessment to the time of death. Subjects who remained alive at study closure were censored at 31 December 2011. Survival status was obtained from national death databases.

\section{Statistical Analyses}

For bivariate analyses, Chi-square tests were performed for categorical variables, log rank test for survival time, Kruskal-Wallis tests for length of stay.

Kaplan-Meier overall survival curves were compared for exposures of interest, namely comorbidity and disability, using two-sided log rank tests. The Cox proportional-hazards regression was implemented to identify predictors of mortality within the cohort. Schoenfeld residuals were used to test the proportionalhazards $(\mathrm{PH})$ assumption after model fitting. In the event of violation of $\mathrm{PH}$ assumption for a specific covariate, the same covariate was included as a stratifying factor in the Cox model. Likelihood ratio tests were used to compare nested models to identify significant predictors of mortality. In the synergistic model, the multiplicative interaction term of comorbidity and disability was included in the model. Potential confounders were adjusted in multiplicative interaction model. All analyses were performed using STATA version 11 (StataCorp LP, USA) with the significance level set at 0.05 .

\section{Results}

19,360 community hospital patient admissions were initially considered. 2,314 (12.0\%) non-rehabilitation were first excluded, followed by $2,604(15.3 \%)$ patients with second or subsequent admissions. A total of 14,442 first rehabilitation admission patients were thus recruited. Among them, 1,638 patients (11.3\%) had missing discharge disability status. The final cohort consisted of 12,804 patients (Figure S1), of whom 4,239 (33.1\%) were alive at the end of the study. The median follow-up time across the cohort was 10.9 years (interquartile range [IQR]: 8.5-13.3 years). The mean age of the cohort at admission was 73.0 (standard deviation [SD]: 11.5) years, mean discharge BI scores was $60.4(\mathrm{SD}=29.0)$, median length of stay was 32.0 days (IQR: 20.0-47.0), and median Charlson comorbidity index score was 3 (IQR: 1-5). Patients who had total disability were more likely to be admitted for stroke $(\mathrm{p}<$ $0.001)$, moderate SES $(\mathrm{p}<0.001)$ and discharged to acute hospital
(25.4\%) compared to other disability groups. Length of stay was not significantly different across the four disability groups $(\mathrm{p}=0.053)($ Table 1).

\section{Bivariate analyses (Table 2)}

Among those who died, significantly more were male $(\mathrm{p}<$ $0.001)$, older $(\geq 65$ years, $\mathrm{p}<0.001)$, widowed $(\mathrm{p}<0.001)$, had a caregiver $(\mathrm{p}<0.001)$, from low or moderate SES group $(\mathrm{p}<0.001)$, had greater comorbidity burden $(\mathrm{p}<0.001)$ and had greater disability $(\mathrm{p}<0.001)$ at discharge, and shorter length of stay $(p=0.005)$ (Table 2, Figure 1). Tests on proportional hazards assumption were performed. Gender, age group (18-64 vs. $\geq 65$ years), year of admission and primary diagnosis at admission violated the proportional hazards assumption. Thus, we stratified the study sample by these variables and obtained pooled estimates. Patients with low, moderate or high comorbidity had $1.43(95 \%$ confidence interval (CI): 1.34-1.54, p<0.001), 1.94 (95\% CI: 1.79-2.09, $\mathrm{p}<0.001)$ and 2.80 (95\% CI: 2.49-3.16, $\mathrm{p}<0.001)$ times mortality risks respectively compared to patients with no comorbidity. Patients with moderate, severe and total disability at discharge had 1.54 (95\% CI: 1.46-1.63, p<0.001), 2.27 (95\% CI: $2.12-2.42, \mathrm{p}<0.001)$ and $2.44(95 \%$ CI: $2.28-2.61, \mathrm{p}<0.001)$ times mortality risks respectively compared to patients with no or mild disability.

\section{Multivariate analyses}

Length of stay was dropped from final model as it became insignificant after adjusting for discharge destination $(\mathrm{p}=0.662)$. Significant predictors of mortality in the final multivariate model were discharge destination, SES group, community hospital and marital status (Table 3). Patients discharged to nursing homes and acute hospitals had 1.14 (95\% CI: 1.05-1.22, p =0.001) and 1.54 (95\% CI: $1.45-1.65, \mathrm{p}<0.001)$ times mortality risk respectively compared to those discharged to home. Patients from moderate or low SES group had 1.12 (95\% CI: 1.03-1.22, $\mathrm{p}=0.007)$ and 1.40 (95\% CI: 1.29-1.53, p<0.001) times greater mortality risk respectively compared to those in high SES group. Patients who were widowed had 1.38 (95\% CI: 1.25-1.53, p < $0.001)$ times mortality risk compared to singles.

The interaction between comorbidity and disability at discharge was significant in the final synergistic interaction model of mortality risk $(\mathrm{p}<0.001)$ (Figure 2, Figure S2). Patients with highest mortality risks had both comorbidity and disability compared to patients with either comorbidity or disability alone 
Table 2. Social demographics by death status at time of censoring and bivariate model of all-cause mortality for hazard ratio.

\begin{tabular}{|c|c|c|c|c|}
\hline & \multirow{2}{*}{$\begin{array}{l}\text { Alive } \\
(n=4239)\end{array}$} & \multirow{2}{*}{$\begin{array}{l}\text { Dead } \\
(n=8565)\end{array}$} & \multirow[t]{2}{*}{ Hazard ratio (95\% Confidence Interval) } & \multirow[t]{2}{*}{ P-value $^{a}$} \\
\hline & & & & \\
\hline Year of admission & & & Stratification & variable \\
\hline 1996 & $112(2.6)$ & $473(5.5)$ & & \\
\hline 1997 & $266(6.3)$ & $1025(12.0)$ & & \\
\hline 1998 & $270(6.4)$ & $1046(12.2)$ & & \\
\hline 1999 & $355(8.4)$ & $1002(11.7)$ & & \\
\hline 2000 & $349(8.2)$ & $856(10.0)$ & & \\
\hline 2001 & $384(9.1)$ & $855(10.0)$ & & \\
\hline 2002 & $365(8.6)$ & $748(8.7)$ & & \\
\hline 2003 & $534(12.6)$ & $834(9.7)$ & & \\
\hline 2004 & $726(17.1)$ & $905(10.6)$ & & \\
\hline 2005 & $878(20.7)$ & $821(9.6)$ & & \\
\hline Primary diagnosis at admission & & & Stratification & variable \\
\hline Stroke & $1708(40.3)$ & $3532(41.2)$ & & \\
\hline Fracture & $1343(31.7)$ & $2438(28.5)$ & & \\
\hline Amputation & $72(1.7)$ & $227(2.7)$ & & \\
\hline Others & 745 (17.6) & $1689(19.7)$ & & \\
\hline Lower limb arthroplasty & $237(5.6)$ & $125(1.5)$ & & \\
\hline Cancer & $47(1.1)$ & $196(2.3)$ & & \\
\hline Falls & $46(1.1)$ & $186(2.2)$ & & \\
\hline Pneumonia & $41(1.0)$ & $172(2.0)$ & & \\
\hline Gender & & & Stratification & variable \\
\hline Female & $2619(61.8)$ & $4844(56.6)$ & & \\
\hline Male & $1620(38.2)$ & $3721(43.4)$ & & \\
\hline Age group & & & Stratification & variable \\
\hline Age: $18-64$ years & $1502(35.4)$ & $1169(13.7)$ & & \\
\hline Age: $\geq 65$ years & $2737(64.6)$ & 7396 (86.4) & & \\
\hline Length of stay (days), median $\left(25^{\text {th }}-75^{\text {th }}\right)$ & $31(20-45)$ & $31(20-47)$ & $0.999(0.998-1.000)$ & 0.010 \\
\hline \multicolumn{5}{|l|}{ Community hospital } \\
\hline A & $1164(27.5)$ & $2076(24.2)$ & 1.00 (ref) & \\
\hline B & $2159(50.9)$ & $4568(53.3)$ & $1.04(0.99-1.10)$ & 0.126 \\
\hline C & $744(17.6)$ & $1637(19.1)$ & $1.06(0.99-1.14)$ & 0.084 \\
\hline D & $172(4.1)$ & $284(3.3)$ & $1.43(1.25-1.63)$ & $<0.001$ \\
\hline \multicolumn{5}{|l|}{ Ethnicity } \\
\hline Chinese & $3738(88.2)$ & 7555 (88.2) & 1.00 (ref) & \\
\hline Malay & $262(6.2)$ & $586(6.8)$ & $1.23(1.13-1.35)$ & $<0.001$ \\
\hline Indian & $186(4.4)$ & $332(3.9)$ & $1.00(0.90-1.13)$ & 0.933 \\
\hline Others & $53(1.3)$ & $92(1.1)$ & $0.89(0.72-1.10)$ & 0.269 \\
\hline \multicolumn{5}{|l|}{ Marital status } \\
\hline Single & $518(12.2)$ & $581(6.8)$ & 1.00 (ref) & \\
\hline Married & $1939(45.7)$ & $3544(41.4)$ & $1.19(1.08-1.30)$ & $<0.001$ \\
\hline Widowed & $1615(38.1)$ & $4238(49.5)$ & $1.47(1.34-1.61)$ & $<0.001$ \\
\hline Separated/Divorced & $167(3.9)$ & $202(2.4)$ & $1.10(0.94-1.30)$ & 0.237 \\
\hline \multicolumn{5}{|l|}{ Caregiver } \\
\hline No & 485 (11.4) & $772(9.0)$ & 1.00 (ref) & \\
\hline Yes & $3754(88.6)$ & $7793(91.0)$ & $1.19(1.10-1.28)$ & $<0.001$ \\
\hline \multicolumn{5}{|l|}{ Socioeconomic status } \\
\hline High & $424(10.0)$ & $783(9.1)$ & 1.00 (ref) & \\
\hline Moderate & $1361(32.1)$ & $3117(36.4)$ & $1.12(1.04-1.22)$ & 0.005 \\
\hline
\end{tabular}


Table 2. Cont.

\begin{tabular}{|c|c|c|c|c|}
\hline & \multirow{2}{*}{$\begin{array}{l}\text { Alive } \\
(n=4239)\end{array}$} & \multirow{2}{*}{$\begin{array}{l}\text { Dead } \\
(n=8565)\end{array}$} & \multirow[t]{2}{*}{ Hazard ratio (95\% Confidence Interval) } & \multirow[t]{2}{*}{ P-value $^{a}$} \\
\hline & & & & \\
\hline Low & 2454 (57.9) & $4665(54.5)$ & $1.31(1.21-1.42)$ & $<0.001$ \\
\hline \multicolumn{5}{|c|}{ Comorbidity burden (Charlson comorbidity index) } \\
\hline None (0) & $1107(26.1)$ & $1270(14.8)$ & 1.00 (ref) & \\
\hline Low (1-3) & $1992(47.0)$ & $3886(45.4)$ & $1.43(1.34-1.54)$ & $<0.001$ \\
\hline Moderate (4-6) & 1049 (24.8) & $2963(34.6)$ & $1.94(1.79-2.09)$ & $<0.001$ \\
\hline High $(\geq 7)$ & $91(2.2)$ & $446(5.2)$ & $2.80(2.49-3.16)$ & $<0.001$ \\
\hline \multicolumn{5}{|c|}{ Discharge disability measured by Barthel Index } \\
\hline No or mild disability $(75-100)$ & $2448(57.8)$ & $2634(30.8)$ & 1.00 (ref) & \\
\hline Moderate disability (50-74) & $1108(26.1)$ & $2642(30.9)$ & $1.54(1.46-1.63)$ & $<0.001$ \\
\hline Severe disability (25-49) & $349(8.2)$ & $1537(18.0)$ & $2.27(2.12-2.42)$ & $<0.001$ \\
\hline Total disability (0-24) & $334(7.9)$ & $1752(20.5)$ & $2.44(2.28-2.61)$ & $<0.001$ \\
\hline \multicolumn{5}{|l|}{ Discharge destination } \\
\hline Home & $3579(86.0)$ & $6195(73.4)$ & 1.00 (ref) & \\
\hline Nursing Home & $291(7.0)$ & $960(11.4)$ & $1.37(1.28-1.47)$ & $<0.001$ \\
\hline Acute hospital & $293(7.0)$ & $1290(15.3)$ & $1.96(1.84-2.09)$ & $<0.001$ \\
\hline
\end{tabular}

aP-value: Cox-proportional hazard model: stratified by age group (18-64, 65 and above), year of admission, gender, primary diagnosis at admission (stroke, fracture, amputation, lower limb arthroplasty, falls, others).

doi:10.1371/journal.pone.0106248.t002

(Figure 2). Among patients who had high comorbidity, patients with no or mild, moderate, severe and total disability had 3.09 (95\% CI: 2.51-3.81, p<0.001), 3.64 (95\% CI: 2.96-4.46, $\mathrm{p}<$ 0.001 ), 5.03 (95\% CI: 3.98-6.35, p<0.001) and 6.57 (95\% CI: 5.15-8.37, $\mathrm{p}<0.001)$ times greater mortality risk respectively compared to those with no comorbidity and no or mild disability

(Table 3, Figure 2).

\section{Discussion}

A national cohort of patients admitted for step-down inpatient rehabilitation after acute hospitalization was followed-up for up to 16 years. Comorbidity, discharge disability, widowhood, low socioeconomic status, readmission into acute care and institutionalization were independent predictors of mortality, reflecting the main elements in models of frailty.[20-24] Our results also demonstrated a novel synergistic effect between comorbidity and discharge disability on long-term mortality.[23,24]

Our results identified predictive variables of mortality that map well onto the frailty cascade model and quantified the increased risks of each of these factors. [4] For example, in the presence of physiological decline (age-related) across multiple physiological systems aggravated by disease processes (comorbidity), an acute physical event (hospitalization) may cause a negative cascade of events from disability, healthcare utilization (readmission and institutionalization) to eventually death. One other integrated

\section{a) Comorbidity burden}

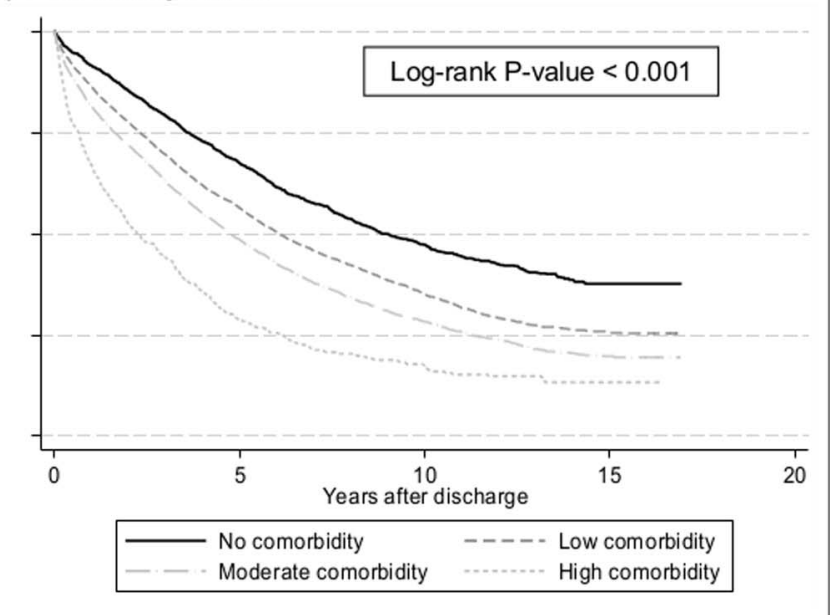

\section{b) Discharge disability}

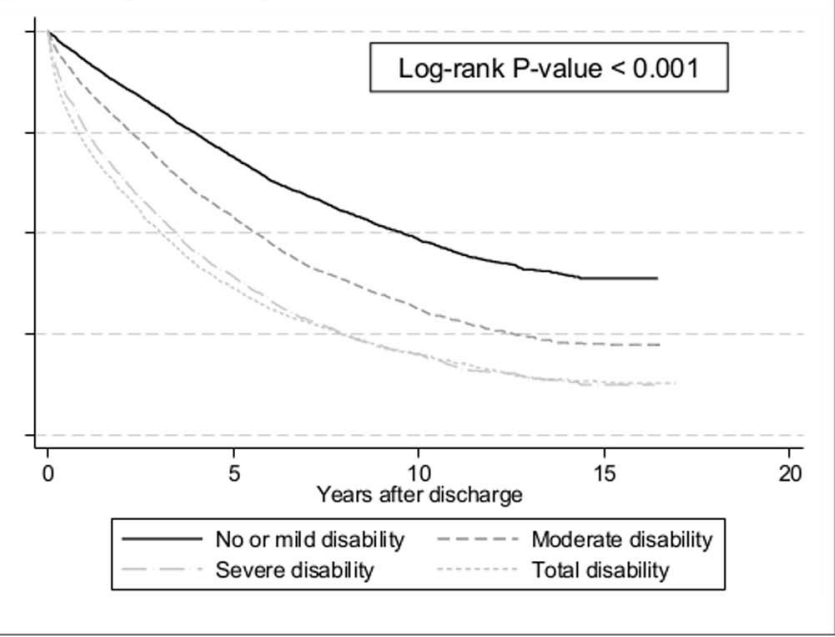

Figure 1. Kaplan-Meier survival curve by comorbidity burden, discharge disability and discharge destination. doi:10.1371/journal.pone.0106248.g001 
Table 3. Multivariate model of all-cause mortality in patients admitted to Singapore community hospitals from 1996 to 2005.

\begin{tabular}{|c|c|c|c|}
\hline & & Hazard ratio ( $95 \%$ Confidence Interval) & P-value \\
\hline Comorbidity $\mathrm{X}$ & Disability & & \\
\hline No & No or mild & 1.00 (ref) & \\
\hline No & Moderate & $1.77(1.55-2.01)$ & $<0.001$ \\
\hline No & Severe & $3.09(2.58-3.71)$ & $<0.001$ \\
\hline No & Total & $2.46(2.01-3.01)$ & $<0.001$ \\
\hline Low & No or mild & $1.47(1.33-1.64)$ & $<0.001$ \\
\hline Low & Moderate & $2.03(1.82-2.26)$ & $<0.001$ \\
\hline Low & Severe & $3.07(2.72-3.46)$ & $<0.001$ \\
\hline Low & Total & $3.22(2.84-3.65)$ & $<0.001$ \\
\hline Moderate & No or mild & $1.99(1.76-2.24)$ & $<0.001$ \\
\hline Moderate & Moderate & $2.69(2.39-3.03)$ & $<0.001$ \\
\hline Moderate & Severe & $3.29(2.89-3.74)$ & $<0.001$ \\
\hline Moderate & Total & $4.42(3.89-5.03)$ & $<0.001$ \\
\hline High & No or mild & $3.09(2.51-3.81)$ & $<0.001$ \\
\hline High & Moderate & $3.64(2.96-4.46)$ & $<0.001$ \\
\hline High & Severe & $5.03(3.98-6.35)$ & $<0.001$ \\
\hline High & Total & $6.57(5.15-8.37)$ & $<0.001$ \\
\hline \multicolumn{4}{|l|}{ Discharge destination } \\
\hline Home & & 1.00 (ref) & \\
\hline Nursing Home & & $1.14(1.05-1.22)$ & 0.001 \\
\hline Acute hospital & & $1.54(1.45-1.65)$ & $<0.001$ \\
\hline \multicolumn{4}{|l|}{ Socioeconomic status } \\
\hline High group & & 1.00 (ref) & \\
\hline Moderate group & & $1.12(1.03-1.22)$ & 0.007 \\
\hline Low group & & $1.40(1.29-1.53)$ & $<0.001$ \\
\hline \multicolumn{4}{|l|}{ Community hospital } \\
\hline A & & 1.00 (ref) & \\
\hline B & & $1.10(1.04-1.17)$ & 0.001 \\
\hline C & & $0.94(0.87-1.02)$ & 0.055 \\
\hline $\mathrm{D}$ & & $1.21(1.05-1.38)$ & 0.007 \\
\hline \multicolumn{4}{|l|}{ Marital status } \\
\hline Single & & 1.00 (ref) & \\
\hline Married & & $1.14(1.03-1.26)$ & 0.009 \\
\hline Widowed & & $1.38(1.25-1.53)$ & $<0.001$ \\
\hline Separated/Divorced & & $1.12(0.95-1.32)$ & 0.190 \\
\hline
\end{tabular}

${ }^{a}$ Cox-proportional hazard model: stratified by age group (18-64, 65 and above), year of admission (1996 to 2005), gender (female, male), primary diagnosis at admission (stroke, fracture, amputation, lower limb arthroplasty, falls, others).

doi:10.1371/journal.pone.0106248.t003

conceptual model of frailty postulated that life course determinants and diseases may lead to frailty and adverse outcomes.[37-39] This model of frailty can also be supported by our findings. For example, widowhood, low socioeconomic status (life course determinants) and comorbidity (diseases) result in disability (physical frailty), institutionalization and death (adverse outcomes). Hence, our results statistically validate earlier theoretical models identifying factors contributing to mortality in frail individuals.

Current models of clinical frailty that combine disability and comorbidity on an additive scale may underestimate the mortality risk, especially in the high-risk groups. The interaction between comorbidity and disability is better considered as a "comorbiditydisability complex". This complex is a central component of many conceptual models of frailty. $[3,4,17,40]$ However, in contrast with current models that assume an additive effect of comorbidity and disability on mortality risk, our findings provide evidence that the effect of this comorbidity-disability complex on mortality risk is synergistic. To our knowledge, no previous study has reported a combined synergistic effect of comorbidity and disability on longterm mortality risk. One approach to understanding the synergistic interaction effect is to tease out how comorbidity and disability impact each other. For example, it can be conceived that comorbidities increase the likelihood of hospitalizations, leading to disability, decreased mobility, sarcopenia, a higher level of frailty, and potentially further comorbidities. These factors are likely to have bidirectional influences and may continuously 


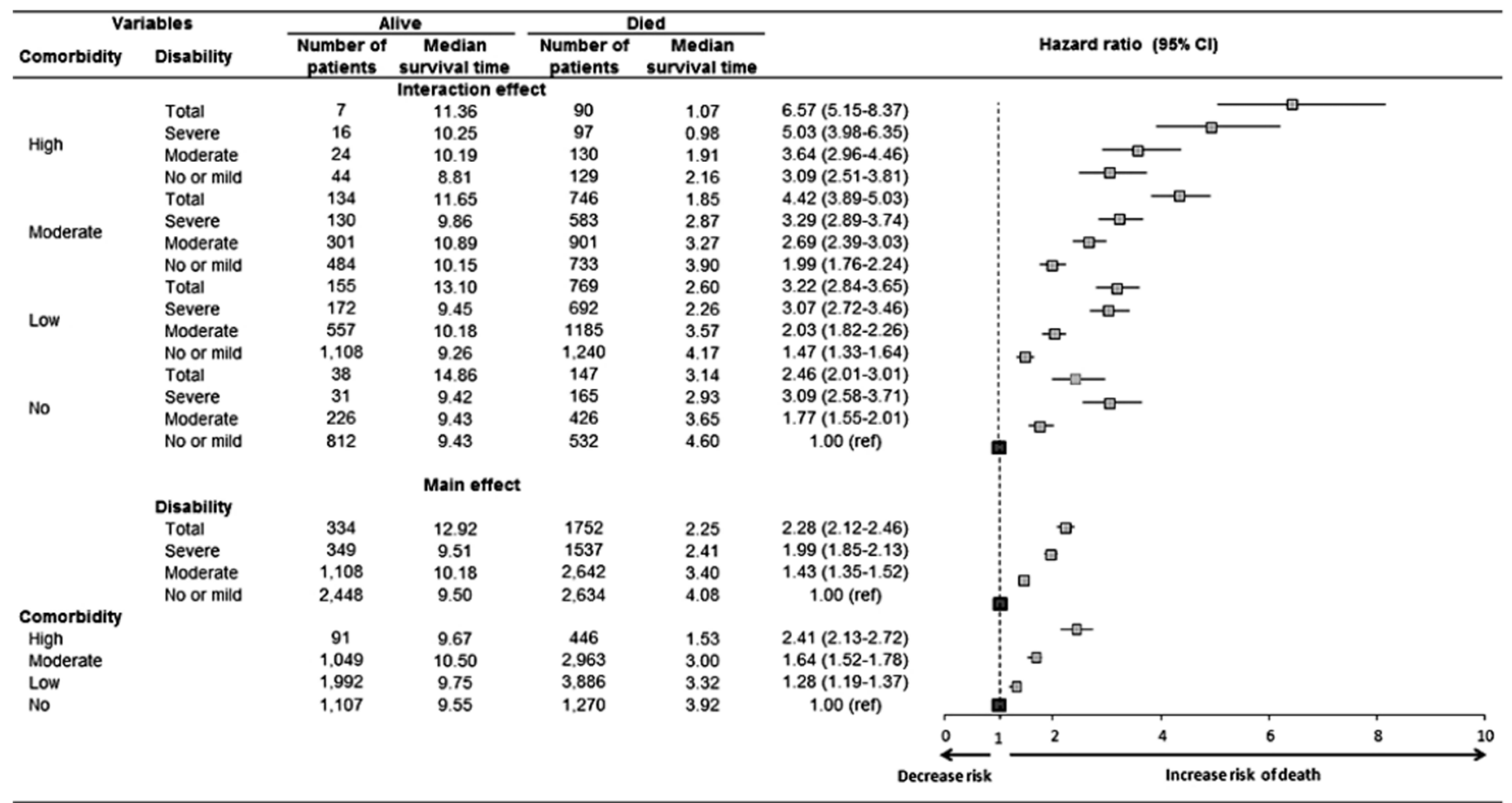

Main effect model: Exposures of interest: comorbidity burden (high, moderate, low and no comorbidity (ref)) and discharge disability (total, severe, moderate and no or mild disability (ref)). Adjusted for fixed effects: discharge destination (nursing home, acute hospital and home (ref)), socioeconomic status (low group, moderate group and high group (ref)), marital status (separated/divorced, widowed, married and single (ref)), and community hospital: A (ref), B, C, D. Stratified by age group (18-64 years, 65 years and above), year of admission (1996 to 2005), gender (female, male), primary diagnosis at admission (stroke, fracture, amputation, lower limb arthroplasty, falls, others)

Interaction effect model: Main effect model + synergistic interaction effect (discharge disability $\mathrm{x}$ comorbidity)

Figure 2. Multiplicative interaction effect of comorbidity and disability in patients admitted to Singapore community hospitals from 1996 to 2005.

doi:10.1371/journal.pone.0106248.g002

propagate in a vicious cycle that perpetuates itself until physical frailty results in premature death.

Clinical management of disability, comorbidity and frailty each has its unique challenges. Disabled older patients are at greater risk of social isolation, institutionalization, and new chronic diseases and initiation of frailty and initially of frailty. [3] Hence, medical care for the disabled involves rehabilitation to maximize function and prevent further decline. Fragmented sub-specialized care focused on single disease leads to complications in patients with multiple comorbidities due to complex relationships between conditions and their treatments.[41] Frail patients also have additional needs beyond those of underlying comorbidity and disability as they are vulnerable to other stressors such as hospitalization, under-nutrition and falls. Additional care is therefore needed to treat pathologic causes of progressive weakness, prevent iatrogenesis and reduce risk factors that result in disability.[3]

\section{Strengths and limitations}

The strengths of our study are a large study sample with long follow-up of up to 16 years. These provided the opportunity to elicit complex long-term relationships such as the synergistic interaction between comorbidity and disability on mortality. The database tracked all patients admitted to community hospitals during the study period and hence was a nationally representative study population. The limitation of this study is the use of retrospective data and incompleteness of records which could have introduced data entry biases. Furthermore, the study was limited to investigating variables that the database included and did not consider other factors may also affect long-term outcomes (e.g. cognition, mental health, quality of life and healthcare decisionmaking). In addition, we excluded 24 patients who died during their hospital stay. As these patients had poorer admission functional scores (mean $=23.9$ vs. 46.3 ) and greater comorbidity burden $($ mean $=4.5$ vs. 2.9) compared to patients in our study, our current risk estimates would be slightly conservative. Finally, the study was completed in an Asian population within a developed economy and an advanced healthcare system. Generalization of these findings to Western and other populations should be done with care.

\section{Conclusions}

This study confirmed that comorbidity and disability are independent predictors of mortality risks in patients after discharge from acute hospitalizations. In addition to widowhood and institutionalization, we also found a novel synergistic interaction effect of the comorbidity-disability complex independent on mortality risk. Future research should consider the feasibility and value of replicating this prospective study in non-Asian populations. The mechanisms through which the comorbidity-disability complex impacts mortality also warrants further investigation. 


\section{Supporting Information}

\section{Figure S1 Flowchart of selection criteria.}

(TIF)

Figure S2 Kaplan-Meier survival curves stratified by comorbidity and discharge disability.

(TIF)

\section{Acknowledgments}

We thank the Board of Directors, management and staff of Ang Mo Kio Thye Hua Kwan Hospital, St Luke's Hospital, St Andrew's Community

\section{References}

1. Morley JE, Vellas B, van Kan GA, Anker SD, Bauer JM, et al. (2013) Frailty consensus: a call to action. J Am Med Dir Assoc 14: 392-397.

2. Rodríguez-Mañas L, Féart G, Mann G, Viña J, Chatterji S, et al. (2013) Searching for an operational definition of frailty: a Delphi method based consensus statement: the frailty operative definition-consensus conference project. J Gerontol A Biol Sci Med Sci 68: 62-67.

3. Fried LP, Ferrucci L, Darer J, Williamson JD, Anderson G (2004) Untangling the concepts of disability, frailty, and comorbidity: implications for improved targeting and care. J Gerontol A Biol Sci Med Sci 59: 255-263.

4. Morley JE, Haren MT, Rolland Y, Kim MJ (2006) Frailty. The Medical clinics of North America 90: 837-847.

5. Covinsky KE, Palmer RM, Fortinsky RH, Counsell SR, Stewart AL, et al. (2003) Loss of Independence in Activities of Daily Living in Older Adults Hospitalized with Medical Illnesses: Increased Vulnerability with Age. Journal of the American Geriatrics Society 51: 451-458.

6. Creditor MC (1993) Hazards of hospitalization of the elderly. Ann Intern Med 118: 219-223.

7. Rudberg MA, Sager MA, Zhang J (1996) Risk Factors for Nursing Home Use After Hospitalization for Medical Illness. The Journals of Gerontology Series A: Biological Sciences and Medical Sciences 51A: M189-M194.

8. Caughey G, Vitry A, Gilbert A, Roughead E (2008) Prevalence of comorbidity of chronic diseases in Australia. BMC Public Health 8: 221.

9. van den Akker M, Buntinx F, Metsemakers JF, Roos S, Knottnerus JA (1998) Multimorbidity in general practice: prevalence, incidence, and determinants of co-occurring chronic and recurrent diseases. J Clin Epidemiol 51: 367-375.

10. Wolff JL, Starfield B, Anderson G (2002) Prevalence, expenditures, and complications of multiple chronic conditions in the elderly. Archives of internal medicine 162: 2269-2276.

11. Fortin M, Bravo G, Hudon C, Vanasse A, Lapointe L (2005) Prevalence of multimorbidity among adults seen in family practice. Ann Fam Med 3:223-228.

12. Charlson ME, Pompei P, Ales KL, MacKenzie CR (1987) A new method of classifying prognostic comorbidity in longitudinal studies: development and validation. J Chronic Dis 40: 373-383.

13. Charlson M, Szatrowski TP, Peterson J, Gold J (1994) Validation of a combined comorbidity index. Journal of Clinical Epidemiology 47: 1245-1251.

14. Rochon PA, Katz JN, Morrow LA, McGlinchey-Berroth R, Ahlquist MM, et al. (1996) Comorbid Illness Is Associated with Survival and Length of Hospital Stay in Patients with Chronic Disability: A Prospective Comparison of Three Comorbidity Indices. Medical Care 34: 1093-1101.

15. Schiller JS, Lucas JW, Peregoy JA. (2012) Summary health statistics for U.S. adults: National Health Interview Survey, 2011. National Center for Health Statistics. Vital Health Stat 10: 67-69.

16. Braithwaite RS, Col NF, Wong JB (2003) Estimating Hip Fracture Morbidity, Mortality and Costs. Journal of the American Geriatrics Society 51: 364-370.

17. Leibson CL, Tosteson ANA, Gabriel SE, Ransom JE, Melton LJ (2002) Mortality, Disability, and Nursing Home Use for Persons with and without Hip Fracture: A Population-Based Study. Journal of the American Geriatrics Society 50: $1644-1650$.

18. Slot KB, Berge E, Dorman P, Lewis S, Dennis M, et al. (2008) Impact of functional status at six months on long term survival in patients with ischaemic stroke: prospective cohort studies. BMJ 336: 376-379.

19. Fried LP, Tangen CM, Walston J, Newman AB, Hirsch C, et al. (2001) Frailty in older adults: evidence for a phenotype. J Gerontol A Biol Sci Med Sci 56: M146-156.

20. Fried LP, Guralnik JM (1997) Disability in older adults: evidence regarding significance, etiology, and risk. J Am Geriatr Soc 45: 92-9100.
Hospital and Bright Vision Hospital for supporting our study and Ministry of Health for providing us with the death data.

\section{Author Contributions}

Conceived and designed the experiments: CG IS HMM GCHK. Performed the experiments: AC NPF KMC BYT EM CHE KKL SM DH GCHK. Analyzed the data: CC BCT SYJT. Contributed reagents/ materials/analysis tools: CC IS HMM BCT NPF SYJT YSN YYT SM DH GCHK. Contributed to the writing of the manuscript: CC IS MHM. Principal investigator of this paper and supervised this study: GCHK. Revised the article critically and gave final approval for publication: GCHK CC IS HMM BCT AC NPF SYJT KMC BYT EM CHE KKL YSN YYT SM DH.

21. Mitnitski A, Song X, Skoog I, Broe GA, Cox JL, et al. (2005) Relative fitness and frailty of elderly men and women in developed countries and their relationship with mortality. J Am Geriatr Soc 53: 2184-2189.

22. Rockwood K, Mitnitski A (2007) Frailty in relation to the accumulation of deficits. The Journals of Gerontology Series A: Biological Sciences and Medical Sciences 62: 722-727.

23. Abellan van Kan G, Rolland YM, Morley JE, Vellas B (2008) Frailty: toward a clinical definition. J Am Med Dir Assoc. United States. pp. 71-72.

24. Van Kan GA, Rolland Y, Bergman H, Morley JE, Kritchevsky SB, et al. (2008) The IANA Task Force on frailty assessment of older people in clinical practice. The Journal of Nutrition Health and Aging 12: 29-37.

25. Gijsen R, Hoeymans N, Schellevis FG, Ruwaard D, Satariano WA, et al. (2001) Causes and consequences of comorbidity: a review. J Clin Epidemiol 54: 661674.

26. Ravindrarajah R, Lee DM, Pye SR, Gielen E, Boonen S, et al. The ability of three different models of frailty to predict all-cause mortality: Results from the European Male Aging Study (EMAS). Archives of Gerontology and Geriatrics.

27. Hubbard RE, Searle SD, Mitnitski A, Rockwood K (2009) Effect of smoking on the accumulation of deficits, frailty and survival in older adults: A secondary analysis from the Canadian study of health and aging. J Nutr Health Aging 13: 468-472.

28. Song X, MacKnight C, Latta R, Mitnitski AB, Rockwood K (2007) Frailty and survival of rural and urban seniors: results from the Canadian Study of Health and Aging. Aging clinical and experimental research 19: 145-153.

29. Ministry of Health $(\mathrm{MOH})$ (2004) Healthcare Services for the Elderly. An information booklet for healthcare professionals.

30. Bernardini J, Callen S, Fried L, Piraino B (2004) Inter-rater reliability and annual rescoring of the Charlson comorbidity index. Advances in peritoneal dialysis Conference on Peritoneal Dialysis 20: 125-127.

31. Shah S, Muncer S (2000) Sensitivity of Shah, Vanclay and Cooper's modified Barthel Index. Clin Rehabil 14: 551-552.

32. Lee J, Reding M (2007) Effects of thiazolidinediones on stroke recovery: a casematched controlled study. Neurochemical research 32: 635-638.

33. Seo NS, Han MS, Lee JS (2006) [Effects of a tilting training program on lower extremities function, depression, and self-efficacy among stroke inpatients]. Taehan Kanho Hakhoe chi 36: 514-522.

34. Shah S, Vanclay F, Cooper B (1989) Improving the sensitivity of the Barthel Index for stroke rehabilitation. J Clin Epidemiol 42: 703-709.

35. Roy CW, Togneri J, Hay E, Pentland B (1988) An Inter-Rater Reliability Study of the Barthel Index. Int J Rehabil Res 11: 67-70.

36. Shinar D, Gross CR, Bronstein KS, Licatagehr EE, Eden DT, et al. (1987) Reliability of the Activities of Daily Living Scale and Its Use in Telephone Interview. Arch Phys Med Rehab 68: 723-728.

37. Gobbens RJJ, van Assen MALM, Luijkx KG, Wijnen-Sponselee MT, Schols JMGA (2010) Determinants of frailty. J Am Med Dir Assoc 11: 356-364.

38. Fried LP, Walston J(2003) Frailty and failure to thrive. Principles of Geriatric Medicine and Gerontology. McGraw-Hill. pp. 1487-1502.

39. Woo J, Leung J (2014) Multi-morbidity, dependency, and frailty singly or in combination have different impact on health outcomes. Age 36: 923-931.

40. Rockwood K, Mitnitski A (2011) Frailty defined by deficit accumulation and geriatric medicine defined by frailty. Clin Geriatr Med 27: 17-26.

41. Redelmeier DA, Tan SH, Booth GL (1998) The treatment of unrelated disorders in patients with chronic medical diseases. N Engl J Med 338: 15161520 . 